\title{
Linguistic validity and reliability of cariogenic food frequency and oral health knowledge, attitude and practice questionnaires for parents of 6-11 years old children in Kota Bharu, Kelantan
}

\author{
Rosnani Ngah ${ }^{1}$, Ruhaya Hasan ${ }^{\text {1* }}$ \& Normastura Abd Rahman ${ }^{1}$ \\ ${ }^{1}$ Department of Dental Public Health, School of Dental Sciences, Universiti Sains \\ Malaysia, 16150 Kubang Kerian, Kelantan Darul Naim, Malaysia
}

\begin{abstract}
Introduction: Cariogenic food intake and oral health knowledge, attitude and practices (KAP) of parents are contributing factors that influence the oral health status of children. As there is lack of a validated questionnaire in Bahasa Melayu (the Malay language) to measure the factors, this cross-sectional study was carried out to establish a linguistically valid and reliable cariogenic food frequency questionnaire (M-CFFQ) and oral health KAP questionnaire (M-OHKAPQ) in Bahasa Melayu, for parents of children aged 6-11 years in Kota Bharu, Kelantan. Methods: Relevant questionnaires in English were selected and translated to Malay language. This process included forward translation, synthesis, backward translation and consolidation to produce the preliminary drafts of M-CFFQ and M-OHKAPQ. Pretesting was conducted on ten parents of children from a primary school in Kota Bharu by face-to-face interview. The findings were reviewed to produce the final versions of M-CFFQ and M-OHKAPQ. A test-retest reliability study was undertaken involving 49 parents. The participants were asked to answer the final M-CFFQ and M-OHKAPQ versions twice, within a 7-14 days interval. Data collected were entered into IBM SPPS version 22 software and analysed using the Intraclass Correlation Coefficient (ICC) test. Results: Semantic, idiomatic, experiential, conceptual as well as content and face validity issues were successfully resolved. Fifteen categories of food/drinks for M-CFFQ and 26 items/questions M-OHKAPQ were produced. The total ICC values achieved for M-CFFQ and M-OHKAPQ were 0.887 and 0.807 , respectively. Conclusion: The M-CFFQ and M-OHKAPQ appeared to be linguistically valid and reliable with excellent test-retest reliability (ICC >0.80).
\end{abstract}

Keyword: Linguistic, cariogenic, oral health, questionnaire, test-retest reliability

\section{INTRODUCTION}

Good oral health in children is essential. In children, it affects their general health (physical, mental, psychological) and social well-being, especially in later life (Schroth, Brothwell \& Moffatt, 2007). Yet, dental caries remains a major public health problem in children worldwide (Peres et al., 2016). Dental caries can be defined as the localised destruction

\footnotetext{
*Corresponding author: Dr Ruhaya Hasan

School of Dental Sciences, Universiti Sains Malaysia, 16150 Kubang Kerian,

Kelantan Darul Naim, Malaysia

Telephone Number: +609-7675751; Fax number: +609-7675505; Email: ruhaya@usm.my

doi: https: / / doi.org/10.31246/mjn-2018-0085
} 
of susceptible dental hard tissues by acidic by-products from the bacterial fermentation of dietary sugar (Fejerskov, 2004). Many children have experienced dental caries as soon as teeth erupt. Petersen et al. (2005) reported that about $60-90 \%$ of school-aged children are affected by dental caries in most industrialised countries in America and the Europe. In the developing countries of Africa and Asia, prevalence rates of dental caries and dental caries experience tend to escalate due to the increasing consumption of sugars and inadequate exposure to fluorides (Petersen et al., 2005). In Malaysia, the latest national oral health survey showed a high prevalence of caries at $71.3 \%$ in 5 years old children and $60.0 \%$ of them had visible plaque (Oral Health Division Malaysia, 2015). In addition, 33.3\% of 12-year old school children had caries on their permanent teeth (Oral Health Division Malaysia, 2017).

Mani et al. (2012) found that the occurrence of caries in children may be influenced by poor dietary habits. In addition, the poor knowledge of oral health, attitude and practices (KAP) of parents contributed to the problem. Children spend much of the time with their parents or primary caregivers when growing up. Most of their early childhood routines such as dietary habits and oral health behaviour are acquired from them at this stage (Naidu \& Davis, 2008). It is crucial for parents to have basic knowledge of diet, nutrition and oral hygiene as a contributory factor to dental caries and to understand their significance, so as to instil good dental practices in their children (Tinanoff $\&$ Reisine, 2009). These include choosing a diet with low or non-cariogenic foods, limiting sweet foods to mealtimes, and brushing teeth after exposures to sugar (Tinanoff \& Reisine, 2009). Parents also play a major role in ensuring the general wellness of their children.
Therefore, the KAP of oral health of parents should impact the oral health of their children. The assessment of these factors is essential for the successful delivery of appropriate oral health knowledge programmes or promotions to the parents. This could help them to understand, create awareness and change their attitudes and practices towards the oral health care of their children.

The Malay language or Bahasa Melayu is the official language for Malaysia. Questionnaires in Bahasa Melayu will benefit the majority of the Malaysian population, cater to respondents who are not proficient in English, particularly those with a lower socio-economic and educational background, and people from rural areas. In addition, there is a lack of an established Bahasa Melayu version of the cariogenic food frequency questionnaire (CFFQ) and oral health knowledge, attitude and practice questionnaire (OHKAPQ) which are linguistically valid and reliable. These may then be used in measuring cariogenic food intake and oral health KAP in the Malaysian population, specifically for parents of preschool and primary school children. Previous local studies had used the translated questionnaires that applied to other age groups, namely secondary school children, infants and toddlers (Cheah et al., 2010; Mani et al., 2010; Zahara et al., 2010: Mani et al. 2012; Ruhaya et al., 2012). The reliability and validity of these questionnaires were only partially reported. On the other hand, designing a new questionnaire may be a demanding, time-consuming and costly task (Epstein, Santo \& Guillemin, 2015). This study was carried out to establish a linguistically valid and reliable Bahasa Melayu version of CFFQ and OHKAPQ questionnaires for parents of children aged 6-11 years old in Kota Bharu, in the state of Kelantan in Malaysia. 


\section{MATERIALS AND METHODS}

\section{Research tools}

The CFFQ used in this study was adapted from Habibian et al. (2001). Initially, it was a food list established from a threeday food diary to describe the dietary habits and oral hygiene behaviour in infants and toddlers over the first 18 months of life, in Mid-Surrey, United Kingdom. It consisted of 18 categories of foods and beverages, namely, all cakes, chocolate, crisps, sweets, sugared cereal, fruits, sugared milk, cow's milk, formula milk, soya milk, formula milk with nonmilk extrinsic sugar (NMES), sugared drinks, sugared beverages, fruit juices, water, drinks no sugar, milk desserts and non-milk dessert. The food list had been adapted in Malaysia by Ruhaya et al. (2012) and Rathmawati et al. (2017) in their studies related to dietary habit and oral health in young children and disabled children. For this study, a formula for food intake frequency was adapted from Chee et al. (1996) and added to the CFFQ. The food intake frequencies were categorised into 7 -point scale rating, in which ' 1 ' represented the never or rarely response, ' 2 ' represented once a month, ' 3 ' represented 2-3 times a month, ' 4 ' represented once a week, '5' represented 2-3 times a week, ' 6 ' represented once daily and '7' represented 2-3 times daily.

Meanwhile, OHKAPQ was adapted from the Oral Health Promotion Evaluation Toolkit by Watt et al. (2004). This toolkit was produced from a project that aimed to critically review, test and publish a set of appropriate age-specific oral health promotion evaluation outcome measures for parents and carers of pre-school children, 12 year-old children and people over 65 years of age in the United Kingdom. For the purpose of this study, 28 questions or outcome measures that are relevant for parents of children aged 6-11 years were selected from the toolkit. These questions were then categorised into three sections, namely, oral health knowledge, attitude and practice in regard to plaque control, fluoride use, diet behaviour and dental attendance.

\section{Process}

This study was conducted from February to May 2017. Ethical approval to conduct this study was obtained from the Human Research Ethics Committee of Universiti Sains Malaysia (JEPem Code: USM/ JEPem/17010067) and National Medical Research Register, Ministry of Health Malaysia (NMRR ID Number: NMRR17-706-35129). It was divided into two phases, namely linguistic validation and test-retest reliability. Figure 1 and Figure 2 show the translation or linguistic validation process and flow charts of both phases.

Phase I: Translation/linguistic validation The linguistic validation process in this study was adapted from the guidelines proposed by Beaton et al. (2000), with slight modifications. Initially, CFFQ and OHKAPQ that were selected underwent a translation process involving forward translation, synthesis, backward translation and consolidation to produce a preliminary Bahasa Melayu version of CFFQ and OHKAPQ which were called M-CFFQ-P and M-OHKAPQ-P. Following the pre-testing stage, ten parents of school children aged 6-11 years old from a primary school in Kota Bharu were selected using quota sampling and invited for a face-to-face interview. The sample size that was decided was based on the process of translation and adaptation guideline recommended by WHO (2001). The inclusion criteria were Malaysian citizens, able to read and write in Bahasa Melayu. All participants gave their consent. During this stage, each participant was informed about the purpose and procedure of the study by a single interviewer. They were asked for their opinions, suggestions and comments regarding the M-CFFQ-P and M-OHKAPQ-P. Any word or expression in these questionnaires that was difficult 
for the participants to comprehend or inappropriately used was probed in detail. A meeting was then held to review and resolve the issues in the pre-test study, to eventually produce the final versions of M-CFFQ and M-OHKAPQ. Members of the expert review committee involved in this phase were a nutritionist specialist, a dental public health specialist, a dental public health (doctorate) student and the researcher.

\section{Phase II: Test-retest reliability}

In test-retest reliability study, the sample size was calculated using a Microsoft Excel spreadsheet file developed by Arifin

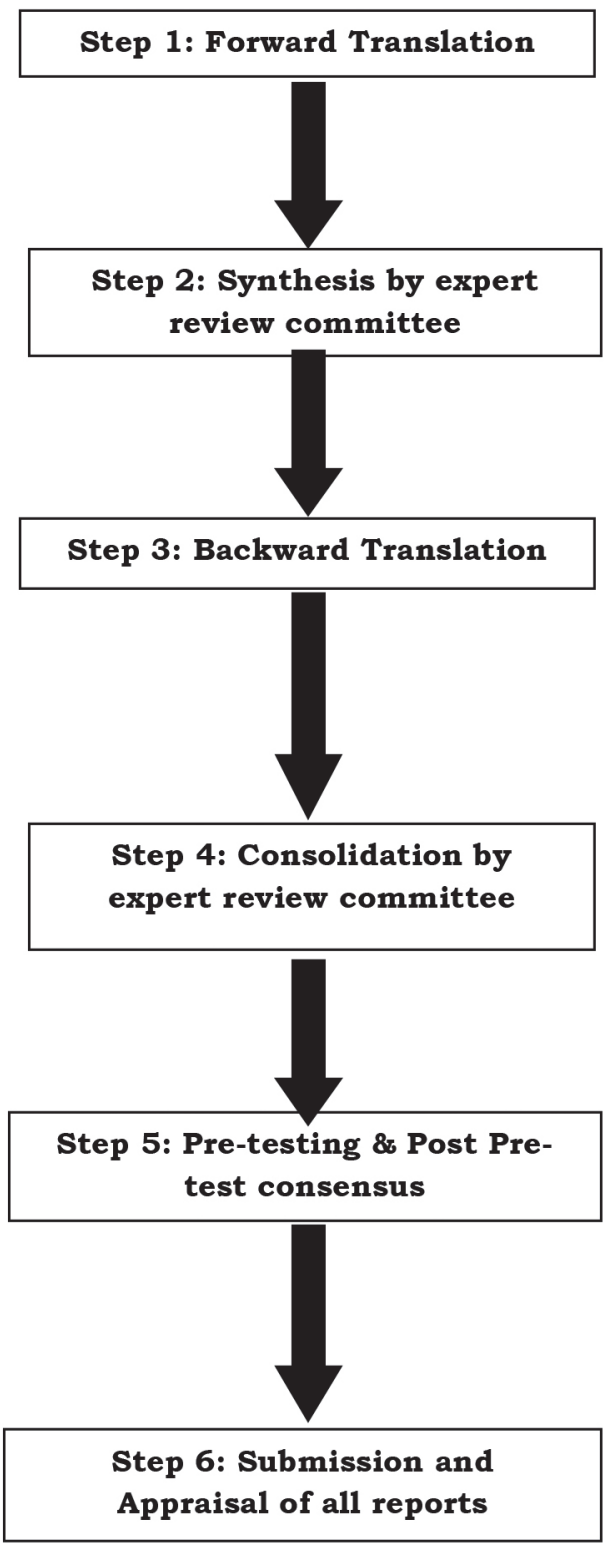

*Two translators for each questionnaire:

T1-CFFQ and T2-CFFQ, T1-OHKAPQ and T2-

OHKAPQ

*Produced two translations for each questionnaire: CFFQ-FT1 and CFFQ-FT2, OHKAPQ-FT1 and OHKAPQ-FT2

*Synthesis of all forward translations

*Resolved discrepancy and produced one common forward for each questionnaire: CFFQFT12 and OHKAPQ-FT12

*Members: nutritionist specialist, dental public health specialist, dental public health (doctorate) student, researcher.

*Two translators for each questionnaire:

T3-CFFQ and T4-CFFQ, T3-OHKAPQ and T4OHKAPQ

*Translated common forward translation CFFQFT12 and OHKAPQ-FT12

${ }^{*}$ Created two back translations for each questionnaire, CFFQ-BT1 and CFFQ-BT2, OHKAPQ-BT 1 and OHKAPQ-BT2

*Reviewed all report

${ }^{*}$ Members: nutritionist specialist, dental public health specialist, dental public health doctorate student, researcher

*Produced preliminary Bahasa Melayu version of CFFQ (M-CFFQ-P) and OHKAPQ (M-OHKAPQ-P)

${ }^{*} n=10$

*parents of total ten schoolchildren aged 6-11 years old in Year 2017, Kubang Kerian 3 National Primary School (quota sampling) *probed to get understanding of the questionnaire to produce final Bahasa Melayu version of CFFQ (M-CFFQ) and OHKAPQ (MOHKAPQ)

*All steps in linguistic validation process had been appropriately reported to verify that the recommended stages were followed.

Figure 1. Flow chart of phase I - linguistic validation process of M-CFFQ and M-OHKAPQ (adapted from a guideline proposed by Beaton et al. (2000) with a slight modification) 


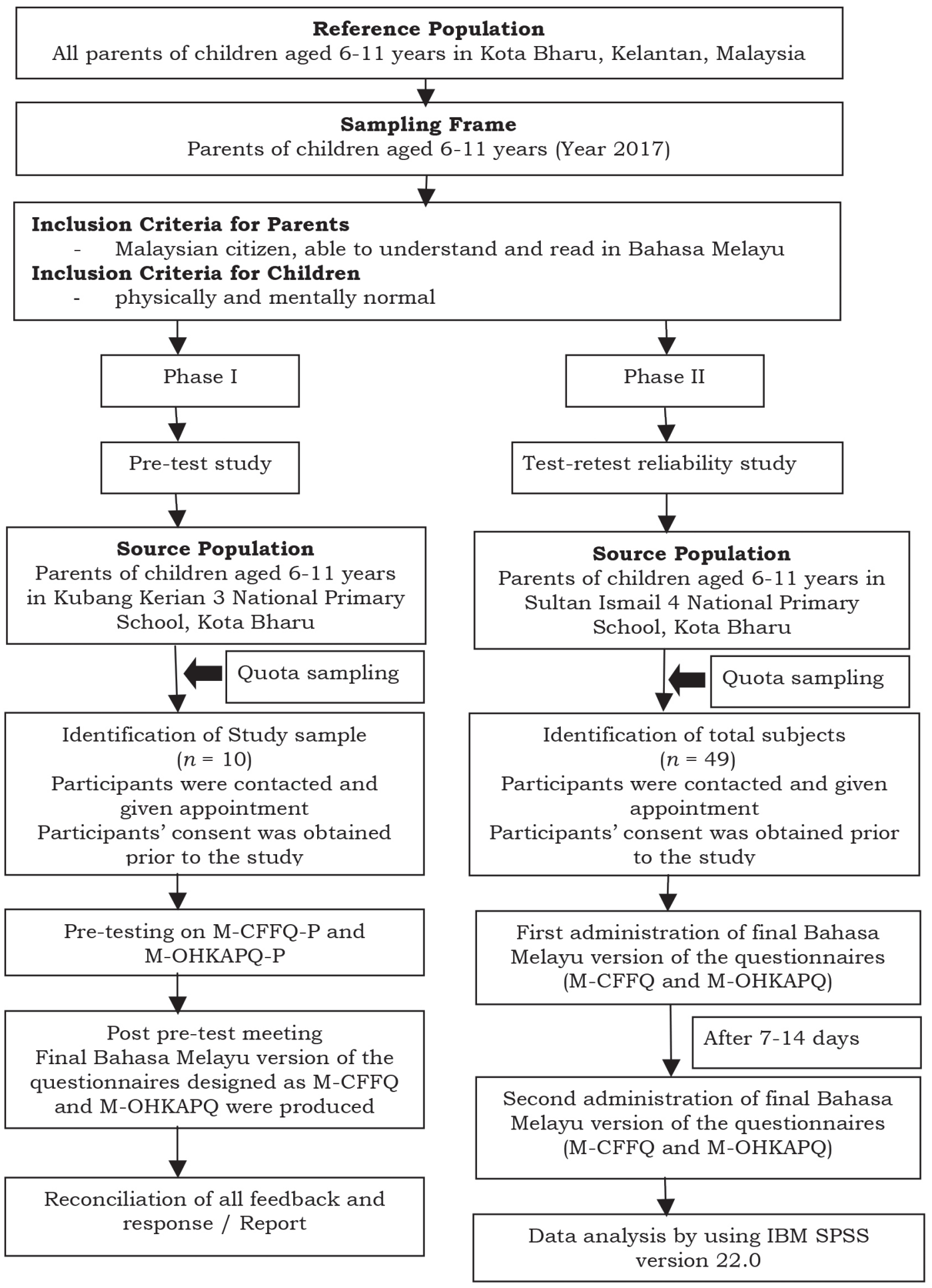

Figure 2. Sampling and flow chart of pre-test study (in phase I) and test-retest reliability study (phase II) 
Table 1. Names of foods and beverages in English (CFFQ) and Bahasa Melayu (M-CFFQ) versions of cariogenic food intake questionnaire

\begin{tabular}{|c|c|c|c|}
\hline No & $\begin{array}{l}\text { English version (CFFQ) } \\
\text { [from Habibian et al. (2001)] } \\
\text { domains and items }\end{array}$ & No & $\begin{array}{l}\text { Bahasa Melayu version (M-CFFQ) } \\
\text { domains and items }\end{array}$ \\
\hline \multirow[t]{3}{*}{1} & $\begin{array}{l}\text { Cake } \\
\text { Cakes, pastries, bun, } \\
\text { breadstick, biscuits }\end{array}$ & 1 & $\begin{array}{l}\text { Kek, Pastri dan Roti } \\
\text { Semua jenis kek, } \\
\text { Semua jenis pastri (contoh : tart, pai, krim } \\
\text { paf, roti canai), } \\
\text { Semua jenis roti dan ban } \\
\text { Lain-lain. Sila nyatakan }\end{array}$ \\
\hline & & 2 & $\begin{array}{l}\text { Biskut } \\
\text { Semua jenis biskut } \\
\text { Lain-lain. Sila nyatakan }\end{array}$ \\
\hline & & 3 & $\begin{array}{l}\text { Kuih tradisional } \\
\text { Semua jenis kuih tradisional } \\
\text { Lain-lain. Sila nyatakan }\end{array}$ \\
\hline 2 & $\begin{array}{l}\text { Chocolates } \\
\text { Including various types of } \\
\text { chocolate bars and wafers }\end{array}$ & 4 & $\begin{array}{l}\text { Coklat } \\
\text { Coklat bar dan biskut bersalut coklat } \\
\text { Lain-lain. Sila nyatakan }\end{array}$ \\
\hline 3 & $\begin{array}{l}\text { Crisps } \\
\text { Crisps and savoury snacks }\end{array}$ & 5 & $\begin{array}{l}\text { Kerepek } \\
\text { Semua jenis kerepek, } \\
\text { Snek berperisa (contoh: makanan ringan atau } \\
\text { jajan) } \\
\text { Lain-lain. Sila nyatakan }\end{array}$ \\
\hline 4 & $\begin{array}{l}\text { Sweets } \\
\text { Chewable sweets, boiled } \\
\text { sweets, lollies }\end{array}$ & 6 & $\begin{array}{l}\text { Gula-gula } \\
\text { Gula-gula boleh kunyah, } \\
\text { Gula-gula keras, } \\
\text { Lolipop } \\
\text { Lain-lain. Sila nyatakan }\end{array}$ \\
\hline 5 & $\begin{array}{l}\text { Sugared cereals } \\
\text { All breakfast cereals, baby } \\
\text { cereal, rusk }\end{array}$ & 7 & $\begin{array}{l}\text { Bijirin bergula } \\
\text { Semua jenis bijirin sarapan bergula } \\
\text { Lain-lain. Sila nyatakan }\end{array}$ \\
\hline 6 & $\begin{array}{l}\text { Fruits } \\
\text { All fresh fruits and dried } \\
\text { fruits }\end{array}$ & 8 & $\begin{array}{l}\text { Buah } \\
\text { Semua jenis buah kering, } \\
\text { Semua jenis jeruk buah, } \\
\text { Semua jenis buah yang disira } \\
\text { Lain-lain. Sila nyatakan }\end{array}$ \\
\hline 7 & $\begin{array}{l}\text { Sugared milk } \\
\text { Milk with added sugar/ } \\
\text { honey (milkshake) }\end{array}$ & 9 & $\begin{array}{l}\text { Susu segar bergula atau susu UHT bergula } \\
\text { Susu segar yang ditambah gula atau madu } \\
\text { Susu UHT yang ditambah gula atau madu } \\
\text { Lain-lain. Sila nyatakan }\end{array}$ \\
\hline 8 & $\begin{array}{l}\text { Soya milk } \\
\text { All soya bean-based milk }\end{array}$ & 10 & $\begin{array}{l}\text { Susu soya } \\
\text { Semua susu berasaskan soya } \\
\text { Lain-lain. Sila nyatakan }\end{array}$ \\
\hline
\end{tabular}


$9 \quad$ Formula milk

All formula milk containing only lactose

10 Formula milk with NMES

All formula milk containing

non-milk extrinsic sugars

11 Sugared drink

Squash,baby drinks, herbal

drinks, fizzy drinks

12 Sugared beverages

Tea with added sugar, coffee with added sugar

13 Fruit juices

All fruit juices and drinks

14 Milk dessert

Yogurt, fromage frais, custard

15 Non-milk dessert

Baby dessert, pureed fruits with added sugar, preserves, honey, table sugar, ice lollies, baby dessert

16 Cow's milk

Cow's milk, goat's milk

17 Water

Water as drink

18 Drinks no sugar

Sugar-free drinks
11 Susu formula dengan gula tambahan

Semua susu formula dengan gula tambahan Lain-lain. Sila nyatakan.

12 Minuman bergula/ susu pekat manis

Minuman bercoklat

Minuman berperisa buah/kordial (contoh:

jagung, laici, anggur, oren dan lain-lain)

Minuman dalam kotak (contoh: teh bunga,

coklat, oren, laici dan lain-lain)

Minuman bergas,

Teh ditambah gula/susu pekat manis (contoh:

teh tarik)

Kopi ditambah gula/susu pekat manis

Minuman herba

Lain-lain. Sila nyatakan.

13 Jus buah-buahan

Semua jus buah-buahan yang;

Tanpa gula

Ditambah gula

Ditambah susu pekat manis

Lain-lain. Sila nyatakan.

14 Pencuci mulut

Semua jenis pencuci mulut

Yogurt dengan gula tambahan

Dadih dengan gula tambahan

Semua jenis aiskrim, cendol, air batu campur.

Lain-lain. Sila nyatakan.

\section{Excluded as not relevant to the content of the} CFFQ

Fresh Cow's and goat's milk are categorised as cariostatic. Meanwhile, water and sugar-free drinks are not cariogenic.

15 Lain-lain

Madu

Semua jenis jem

Kaya

Semua jenis colek/ sos/ kicap (untuk buah, rojak, lok chen, keropok dan lain-lain)

Lain-lain. Sila nyatakan 
(2015), based on a formula designed by Walter, Eliasziw and Donner (1998). The lower limit of accepted reliability $\left(\mathrm{P}_{0}\right)$ was set at 0.6, whereas expected reliability coefficient $\left(\mathrm{P}_{1}\right)$ was decided at 0.8 (Zulkifli, Tin-Oo \& Saddki, 2015), with $80 \%$ power and alpha 0.05. The sample size obtained was 39 subjects. After assuming a 20\% drop-out rate, the sample size was increased to 49 subjects. Thus, 49 eligible parents of children aged 6-11 years of age were recruited via quota sampling from another primary school in Kota Bharu. The selected parents were contacted and asked to complete the M-CFFQ and M-OHKAPQ forms twice with 7-14 days intervals between the first and second administration of the questionnaires. Appointments were made with the parents and the interviews conducted accordingly. All participants gave their informed consent. Data collected in this study was entered into the IBM SPPS version 22 software. The intraclass Correlation Coefficient (ICC) test was used to check for test-retest reliability by assessing the agreement of the responses in the time interval. The level of significance was set at $p<0.05$.

\section{RESULTS}

\section{Phase I: Linguistic validation}

The Bahasa Melayu versions of CFFQ (M-CFFQ) and OHKAPQ (M-OHKAPQ) showed satisfactory equivalence with the respective English versions of CFFQ (Table 1) and OHKAPQ (Table $2)$. Some modifications were made by the expert committee to achieve better semantic, idiomatic, experiental as well as conceptual equivalences. Meanwhile, content validity and face validity of M-CFFQ and M-OHKAPQ were obtained through expert committee review in the synthesis, consolidation and post pretest stages. Some domains and items in $\mathrm{M}-\mathrm{CFFQ}$ and $\mathrm{M}-\mathrm{OHKAPQ}$ required modifications to suit common Malaysian cariogenic foods/drinks and to be properly translated into local language and culture. Common Malaysian foods / drinks include traditional delicacies, spicy and sweetened soy bean sauce (colek), coconut-spread (sekaya or kaya), tea drinks with sweetened creamer (teh tarik), and cendol which is an icy dessert with glutinous pellets, coconut milk, palm sugar, and sometimes with added coloured syrup, sweet corn and black beans. None of the intended objectives and concept of the questionnaires was compromised in the process. At the end of this phase, 15 categories of foods/ drinks for M-CFFQ and 26 items/ questions for M-OHKAPQ were produced. Sociodemographic profile of participants in the pre-test stage is shown in Table 3.

\section{Phase II: Test-retest reliability}

The socio-demographic profile of respondents in test-retest reliability is shown in Table 3.

Table 4 shows the ICC analysis of M-CFFQ and M-OHKAPQ for each domain. Total ICC value of $\mathrm{M}-\mathrm{CFFQ}$ and M-OHKAPQ are 0.887 and 0.807 , respectively. Using the guideline of interpretation of the ICC by Cicchetti (1994), these findings indicate that M-CFFQ and M-OHKAPQ showed excellent agreement between test and retest study and both are stable between the two different occasions or time.

\section{DISCUSSION}

\section{Phase I: Translation/Linguistic validation and equivalences}

Both M-CFFQ and M-OHKAPQ achieved semantic, idiomatic, experiental and conceptual equivalences as in original sources through the translation/ linguistic validation process (Karthikeyan, Manoor \& Supe, 2015; Beaton et al., 2000). The content validity and face validity of the questionnaires were assessed, as suggested by Karthikeyan et al. (2015). Amendments in both questionnaires were made in accordance with the comments received 
Table 2. Questions in English (OHKAPQ) and Bahasa Melayu (M-OHKAPQ) versions of KAP questionnaire

\begin{tabular}{ll}
\hline No $\quad$ English Version (OHKAPQ) \\
\hline 1 & Section: Knowledge \\
& Sugary snacks / drinks are best \\
limited to meal times. & \\
& Yes \\
No & \\
Do not know \\
If your child had a bag of sweets, \\
would it be better for his / her teeth \\
to eat them \\
All in one go \\
A few now and rest later \\
Do not know
\end{tabular}

3 Fruit drinks designed specially for children are safe to teeth

$$
\begin{aligned}
& \text { Yes } \\
& \text { No } \\
& \text { Do not know }
\end{aligned}
$$

$4 \quad$ Most medicines are sugar-free

Yes
No
Do not know

$5 \quad$ At what age do you think that you should start to brush your child's teeth?

At birth

When first teeth come through

1 - 2 years

Over 2 years

Do not know

$6 \quad$ You should brush your child's teeth after each meal to prevent decay

Yes

No

$$
\text { Do not know }
$$

$7 \quad$ How often each day should children's teeth to be brushed?

Once

Twice

Three times

Never

Do not know
Bahasa Melayu version OHKAPQ (M-OHKAPQ)

Bahagian: Pengetahuan

Makanan/minuman bergula paling sesuai dihadkan pada waktu makan utama (sarapan, makanan tengahari dan malam) sahaja

Ya

Tidak

Tidak tahu

Jika anak anda mempunyai sejumlah gula-

gula (satu peket atau sebungkus), adalah lebih baik untuk mereka sekiranya dimakan:

Semua sekaligus

Sedikit demi sedikit

Tidak tahu

Minuman berperisa buah-buahan (contoh:

laici, anggur, oren dan lain-lain) adalah

selamat untuk gigi anak anda

Ya

Tidak

Tidak tahu

Kebanyakan ubat-ubatan dalam bentuk cecair mengandungi gula.

$$
\text { Ya }
$$

Tidak

Tidak tahu

Pada pendapat anda bilakah umur yang paling sesuai untuk mula menjaga kebersihan mulut anak anda?

Selepas lahir

Apabila gigi pertama telah tumbuh

1-2 tahun

Lebih dua tahun

Tidak tahu

Anak-anak perlu memberus gigi atau berkumur setiap kali selepas makan untuk mengelakkan kerosakan gigi.

Ya

Tidak

Tidak tahu

Berapa kali sehari gigi anak anda perlu diberus?

Sekali

Dua kali

Tiga kali

Tidak pernah

Tidak tahu 
$8 \quad$ How much toothpaste should be used for each brushing of a child's teeth?

Blob, the size of a pea

$1 / 2$ brush head length

Whole brush head length

Does not matter

Do not know

9 Fluoride strengthens tooth enamel

True

False

Do not know

10 At what age should you start taking your child to the dentist?

$0-12$ months

13-24 months

25-36 months

When at school

When they have a toothache

Do not know

11 For what reason should you take your child to the dentist?

Because they have a toothache

For a checkup

To get them used to go

Do not know

Others

Section: Attitude

12 There is no need to be concerned about baby teeth.

Strongly agree

Agree

Undecided

Disagree

Strongly Disagree

13 It is not worthwhile to get cavities in baby teeth filled because those teeth fall out anyway

Strongly agree

Agree

Undecided

Disagree

Strongly Disagree

14 Regular visits to the dentist are very important.

Strongly agree

Agree

Undecided

Disagree

Strongly Disagree
Berapa banyak ubat gigi yang diperlukan setiap kali memberus gigi anak anda?

Sekecil saiz kacang pis

Setengah saiz kepala berus gigi

Keseluruhan saiz kepala berus gigi

Tidak penting.

Tidak tahu

Florida menguatkan enamel (permukaan luar) gigi.

Betul

Salah

Tidak tahu

Pada umur berapakah anda perlu mula membawa anak berjumpa doktor gigi?

0-12 bulan

13-24 bulan

25-36 bulan

Apabila mula bersekolah

Apabila sakit gigi

Tidak tahu

Mengapakah anda perlu membawa anak berjumpa dengan doktor gigi?

Sebab sakit gigi

Untuk pemeriksaan

Untuk membiasakan anak

Tidak tahu

Lain-lain

Bahagian: Sikap

Adalah perlu untuk mengambil berat

berkenaan gigi susu.

Sangat setuju

Setuju

Tidak pasti

Tidak setuju

Sangat tidak setuju

Adalah berbaloi menampal gigi susu yang berlubang walaupun gigi tersebut akan diganti dengan gigi kekal.

Sangat setuju

Setuju

Tidak pasti

Tidak setuju

Sangat tidak setuju

Pemeriksaan berkala oleh doktor gigi adalah sangat penting

Sangat setuju

Setuju

Tidak pasti

Tidak setuju

Sangat tidak setuju 
15 If your child had a bad back tooth and it was not a baby tooth, but a second tooth, would you rather it was filled or taken out?

Filled

Taken out

Do not know

16 If your child had a bad front tooth and it was not a baby tooth, but a second tooth, would you rather it was filled or taken out?

Filled

Taken out

Do not know

17 Brushing my teeth twice a day for 2 -3 minutes will keep the gums health

Extremely likely

Likely

Neither likely nor unlikely

Unlikely

Extremely likely

18 Some children insist on brushing their own teeth from a very early age. Does your child:

Brush his/ her own teeth? Has it done for him/her (adult always assists and repeats)? Sometimes do it alone and sometimes have it done for him/her?

Section: Practice

How often does your child brush his/her teeth or have them brushed?

Less often than once a week

At least once a week but not every day

Once a day

Twice a day

More than twice a day

20 Has your child started using toothpaste?

Yes

No

(If 'Yes', answer Q21 onwards) (If 'No', the questionnaire ends here)
Adakah anda mahu jika gigi geraham kekal anak anda yang rosak untuk.......?

\section{Ditampal}

Dicabut

Tidak tahu

Adakah anda mahu jika gigi depan kekal anak anda yang rosak untuk......?

\section{Ditampal \\ Dicabut \\ Tidak tahu}

Memberus gigi dua kali sehari selama 2-3 minit dapat mengekalkan kesihatan mulut, gigi dan gusi.

Sangat setuju

Setuju

Tidak pasti

Tidak setuju

Sangat tidak setuju

Sesetengah anak-anak hendak memberus gigi sendiri di usia awal kanak-kanak. Adakah anak anda

Memberus giginya sendiri?

Memberus gigi dengan dibantu orang dewasa?

Ada masanya memberus sendiri dan ada masa dibantu orang dewasa

Bahagian: Amalan

Berapa kerap anak anda memberus gigi?

Kurang dari sekali seminggu

Sekurang-kurangnya sekali seminggu tetapi tidak setiap hari

Sekali sehari

Dua kali sehari

Lebih dari dua kali sehari

Adakah anak anda telah mula menggunakan ubat gigi?

Ya

Tidak

Jika Ya, sila jawab Soalan 21dan seterusnya. Jika Tidak, sila jawab Soalan 23 dan seterusnya. 
21 Does the toothpaste your child uses contain fluoride?

Yes

Do not know

22 The amount that my child usually use during tooth brushing :

Blob, the size of a pea

$1 / 2$ brush head length

Whole bush head length

Does not matter

Do not know

23 What drink do you usually give to your child before bed or during the night?

Tap/still mineral water

Fizzy mineral water (not

flavoured)

Fizzy mineral water(flavoured)

Herbal drink

Milk drinks (not flavoured)

Flavoured milk (eg: Horlicks,

Ovaltine, milkshake)

Non-diluted fruit juice

Fruit drink/squash

Diluted fruit juice

Fizzy drink

Tea or coffee

Something else

Do not know

If yes, answer Q24 onwards

If never, Stop here

24 Nowadays, how often does your child take something to drink before bed or during the night?

Every night

4-6 nights a week

1-3 nights a week

Less often than once a week

Never

25 How often does your child have something to eat between meals during the day?

Most of the time

Some of the time

Once in the while

Rarely

Never

Do not know
Adakah ubat gigi anak anda mengandungi fluorida?

Ya

Tidak

Tidak tahu

Semasa memberus gigi anak saya menggunakan ubat gigi......?

Sekecil saiz kacang pis

Setengah saiz kepala berus gigi

Keseluruhan saiz kepala berus gigi

Tidak penting

Tidak tahu

Apakah minuman yang sering anak anda ambil sebelum tidur atau pada waktu malam?

Jika ya untuk pilihan jawapan ini, sila jawab

Soalan 24 dan seterusnya

Susu dengan gula tambahan

Minuman bercoklat

Jus buah-buahan

Minuman berperisa buah-buahan

(contoh laici, oren, anggur dan lain-lain)

Minuman dalam kotak (contoh : teh bunga,

coklat, anggur dan lain-lain)

Minuman bergas

Teh/kopi dengan susu pekat manis atau gula

Minuman herba

Lain-lain. Sila nyatakan:

Jika ya untuk pilihan jawapan ini, sila terus

ke soalan 25 dan seterusnya

Air kosong/mineral

Tidak tahu

Tidak minum

Berapa kerap anak anda minum minuman tersebut dalam tempoh seminggu?

Setiap malam

4-6 malam

1-3 malam

Kurang dari sekali

Berapa kerap anak anda makan makanan bergula di antara waktu makan utama dalam sehari? (makan utama: sarapan, makan tengahari dan malam)

Pada kebanyakan masa

Beberapa kali dalam sehari

Sekali dalam sehari

Jarang-jarang

Tidak pernah

Tidak tahu 


\begin{tabular}{|c|c|c|}
\hline 26 & $\begin{array}{l}\text { How often does your child have } \\
\text { something to eat between meals in } \\
\text { the evening or in weekend? }\end{array}$ & $\begin{array}{l}\text { Berapa kerap anak anda makan makanan } \\
\text { bergula di antara waktu makan utama pada } \\
\text { hujung minggu? } \\
\text { (makan utama: sarapan, makan tengahari dan } \\
\text { malam) }\end{array}$ \\
\hline & Most of the time & Pada kebanyakan masa \\
\hline & Some of the time & Beberapa kali dalam sehari \\
\hline & Once in the while & Sekali dalam sehari \\
\hline & Rarely & Jarang-jarang \\
\hline & Never & Tidak pernah \\
\hline & Do not know & Tidak tahu \\
\hline
\end{tabular}

Table 3. Socio-demographic profile of the respondents

\begin{tabular}{|c|c|c|}
\hline Variables & $\begin{array}{c}\text { Pre-test stage } \\
(n=10)\end{array}$ & $\begin{array}{l}\text { Test Retest Reliability } \\
(n=49)\end{array}$ \\
\hline \multicolumn{3}{|l|}{ Respondents, n (\%) } \\
\hline Fathers & $2(20.0)$ & $9(18.4)$ \\
\hline Mothers & $8(80.0)$ & $40(81.6)$ \\
\hline \multicolumn{3}{|l|}{ Sex of child, $n(\%)$} \\
\hline Boy & $4(40.0)$ & $24(49.0)$ \\
\hline Girl & $6(60.0)$ & $25(51.0)$ \\
\hline \multicolumn{3}{|l|}{ Age (years), Mean \pm SD) } \\
\hline Child & $8.5 \pm 1.6$ & $8.6 \pm 1.8$ \\
\hline Respondent & $40.4 \pm 5.9$ & $40.2 \pm 6.4$ \\
\hline \multicolumn{3}{|l|}{ Respondent's race, $\mathrm{n}(\%)$} \\
\hline Malay & $10(100.0)$ & $49(100.0)$ \\
\hline Chinese & $0(0.0)$ & $0(0.0)$ \\
\hline Indian & $0(0.0)$ & $0(0.0)$ \\
\hline Others & $0(0.0)$ & $0(0.0)$ \\
\hline \multicolumn{3}{|l|}{ Respondent's education level, n (\%) } \\
\hline No formal education & $0(0.0)$ & $0(0.0)$ \\
\hline Primary school & $0(0.0)$ & $0(0.0)$ \\
\hline Lower secondary school & $1(10.0)$ & $3(6.1)$ \\
\hline Upper secondary school & $5(50.0)$ & $11(22.5)$ \\
\hline Diploma or equivalent & $1(10.0)$ & $11(22.5)$ \\
\hline Degree/Master/PhD & $2(20.0)$ & $24(48.1)$ \\
\hline $\begin{array}{l}\text { House income per month (Ringgit } \\
\text { Malaysia), Median (IQR) }\end{array}$ & $1100(2600)$ & $5000(6100)$ \\
\hline \multicolumn{3}{|l|}{ Oral health information sources, $\mathrm{n}(\%)$} \\
\hline None & $0(0.0)$ & $0(0.0)$ \\
\hline Doctor/Nurses/ Healthcare workers & $8(80.0)$ & $37(75.5)$ \\
\hline Television/Radio & $8(80.0)$ & $29(59.2)$ \\
\hline Newspaper/Article & $9(90.0)$ & $29(59.2)$ \\
\hline Internet & $8(80.0)$ & $31(63.3)$ \\
\hline Family/Friend & $7(70.0)$ & $24(49.0)$ \\
\hline Others & $0(0.0)$ & $0(0.0)$ \\
\hline
\end{tabular}


Table 4. Intraclass Correlation Coefficient (ICC) of M-CFFQ and M-OHKAPQ

\begin{tabular}{clcc}
\hline \multirow{2}{*}{ No } & \multicolumn{1}{c}{ Domains } & $\begin{array}{c}\text { Test-Retest Reliability } \\
(\text { ICC) }\end{array}$ & 95\% CI \\
\hline M-CFFQ & & & \\
1 & Cake & 0.719 & $0.542,0.834$ \\
2 & Biscuits & 0.803 & $0.676,0.884$ \\
3 & Traditional delicacies & 0.608 & $0.400,0.758$ \\
4 & Chocolate & 0.729 & $0.550,0.841$ \\
5 & Crisps & 0.768 & $0.624,0.862$ \\
6 & Sweets & 0.801 & $0.669,0.883$ \\
7 & Sugared cereals & 0.872 & $0.785,0.926$ \\
8 & Fruits & 0.663 & $0.472,0.794$ \\
9 & Sugared fresh /UHT milk & 0.687 & $0.504,0.811$ \\
11 & Formula milk with added & 0.815 & $0.694,0.892$ \\
10 & sugar & & \\
12 & Soya milk & 0.703 & $0.530,0.821$ \\
13 & Sugared/Sweetened drink & 0.699 & $0.488,0.826$ \\
14 & Fruits Juice & 0.767 & $0.622,0.862$ \\
15 & Dessert & 0.718 & $0.550,0.830$ \\
Total ICC Value of M-CFFQ & 0.866 & $0.775,0.922$ \\
M-OHKAPQ & Others & 0.887 & $0.788,0.938$ \\
1 & Knowledge (K) & & \\
2 & Attitude (A) & & $0.542,0.829$ \\
Total ICC Value & Practice (P) & 0.714 & $0.759,0.918$ \\
\hline
\end{tabular}

${ }^{\dagger}$ ICC was calculated using two way mixed model with absolute agreement type option (IBM SPPS version 22)

from the participants, taking into consideration the characteristics and socio-demographic profile of the target population (Table 3) can influence the feasibility, readability, consistency, formatting, and clarity of language (Vyas et al., 2016).

\section{Content validity}

Content validity is defined as the extent to which a measurement reflects the specific intended domain of content. The evidence for content validity was obtained from previous literature, the views of experts and from local target groups, as there are no objective measurements to test content validity (Laaksonen, 2012). With regard to $\mathrm{M}-\mathrm{CFFQ}$, there were some issues encountered during forward translation, concerning particular words and items that are uncommon in Malaysian population or which could not be translated into Bahasa Melayu. Otherwise, the words and items were translated into terms that had similar meaning without compromising the content. These included words such as 'breadstick' which was replaced with 'bread' (roti) and 'squash' which was redefined as 'fruit flavoured drinks' (minuman berperisa buah-buahan). However, 'fromage fraise' and 'baby dessert' items were excluded from M-CFFQ as these foods were not familiar or relevant to these preschool and primary school children. These changes 
were consistent with those of ShingaIshihara et al. (2014) who had excluded the items 'applesauce' and 'breakfast drinks' from their FFQ which was adapted from a western study, as these were not familiar to the Japanese.

In our study, the expert committee also agreed to omit the items that were not relevant to the intended content for the M-CFFQ. These were the 'water' and 'drinks no sugar' domains, which were identified as not cariogenic. In addition, the domains on 'cow's milk' and 'formula milk that contains only lactose' were excluded based on the report by RuggGunn and Woodward (2011) which stated that these types of milk are virtually noncariogenic. The sugar present is lactose, which is the least cariogenic of all monoand disaccharides. Milk also contains calcium phosphate, fat and casein that protect against demineralisation of enamel and overcome the cariogenicity of the milk (Rugg-Gunn \& Woodward, 2011). Furthermore, Rugg-Gunn and Woodward (2011) concluded that milk and milk products such as cheese and yoghurt without added sugars were safe and possibly beneficial for teeth. Therefore, the item 'yoghurt' in the CFFQ was substituted with yoghurt with added sugar' in the M-CFFQ as the latter is better in representing the intended content of the questionnaire.

In addition, the item 'fresh fruit' in the original CFFQ was omitted since its cariogenicity is considered very low, unless it was consumed at least 17 times per day (Moynihan \& Petersen, 2004). Besides, data from epidemiological studies largely showed that fresh fruit is non-cariogenic and its consumption had been reported to have a negative correlation with dental caries (Arora \& Evans, 2012). However, the consumption of fresh fruits is encouraged by Malaysian Dietary Guidelines and health reports from Australia recommend at least two servings of fresh fruits per day (NCCFN, 2010; Arora \& Evans, 2012). Fruit consumption is also supported by
The Eatwell Plate, the United Kingdom national food guide that recommends that one-third of dietary volume should be provided by fruits and vegetables (Arora \& Evans, 2012). The general opinion appears to favour the consumption of whole fruits, as they benefit overall health and stimulate salivary flow (Arora \& Evans, 2012).

In order to include more potential cariogenic foods in Malaysian population, our expert committee decided to add the domains, 'traditional delicacies' and 'others' as they captured miscellaneous local foods such as 'honey', 'coconut spread' (sekaya or kaya), and 'sweet sauce' (colek). The items 'sugared/ sweetened drinks' and 'packet drinks' (minuman dalam kotak) were introduced as these were commonly available in retail outlets. This, again, is in agreement with Shinga-Ishihara et al. (2014), who added five new items of traditional fresh and semi-dry confectioneries that are common in Japan to their adapted food questionnaire. These were bread filled with bean jam or fruit jam (An-pan or Jampan), rice cracker (Senbei), bun with a bean-jam filling (Manju), rice cake (Mochi), and bar of sweet jellied adzukibean paste (Youkan).

As for M-OHKAPQ, there was a challenge in defining, in Bahasa Melayu, 'often', which means 'kerap' (frequent) or 'selalu' (always or consistent) and other terms related to the frequency measurement such as 'some of the time', 'once in a while' and 'rarely'. However, the expert committee managed to address these issues by using suitable local terms. The same idea was applied by Hammond et al., (2014) who undertook the linguistic validation of the Evaluation of Daily Activity Questionnaire (EDAQ) for use in rheumatoid arthritis from the Swedish language to British-English. They made several changes in the forward translation, for example, for 'high' and 'low spirited.' As these terms are not in everyday use in British-English and they decided to replace them with 'happy' and 
'unhappy', respectively (Hammond et al., 2014).

Otherwise, there were minor changes regarding the use of appropriate nouns, words and expression as well as rearrangement of the questions in M-CFFQ and M-OHKAPQ. These were done for better understanding and, eventually, to increase content validity (Shinga-Ishihara et al., 2014).

\section{Face validity}

Face validity of the M-CFFQ and M-OHKAPQ was achieved through pre-test and test-retest reliability. The main purpose of pre-testing was to determine whether all the items were comprehensive and acceptable. In this study, cognitive interview/debriefing approach was used to assess whether the concepts, questions and response alternatives were clearly conveyed to the participants by the questionnaires, as intended (Laaksonen, 2012). Generally, there were no serious difficulties encountered by the participants in understanding the words and sentences used in the M-CFFQ and M-OHKAPQ.

Minor amendments and modifications were done following the pre-test study. Some of the participants suggested that certain domains and items in M-CFFQ and M-OHKAPQ should provide a few examples or explanation for better understanding. This involved the items 'pastries', 'snack' and 'sugared milk' (susu bergula) in the M-CFFQ and the terms 'snack', 'main meal', 'enamel' and 'fruit drinks' in the M-OHKAPQ. This matter was successfully resolved by the expert committee by adding the examples as suggested by the participants. On other hand, some phrases that could potentially cause confusion were substituted with other terms with a similar meaning. These included 'a bag of sweets', replaced with 'sepeket atau sebungkus gula-gula', 'a few now and the rest later' (makan sedikit demi sedikit), 'after meals' (selepas makan), and 'blob, the size of a pea' (sekecil saiz kacang pis).
Our undertaking of the face validity of M-CFFQ and M-OHKAPQ was in line with that of study of Hammond et al. (2014). They received recommendations from their cognitive debriefing participants that requested simplification of some part of the adapted questionnaire, combining items where possible and deleting uncommon items. There were also suggestions to add relevant items and to omit some inappropriate items which were not relevant to their target group. All changes were made through a consensus approach (Hammond et al., 2014). The words and phrases used in M-CFFQ and M-OHKAPQ were carefully chosen to avoid any misunderstanding. Content validity was again obtained through post pre-test meeting of the experts to resolve the issues that were identified during the face validation process.

\section{Test-retest reliability}

Test-retest reliability of $\mathrm{M}-\mathrm{CFFQ}$ and M-OHKAPQ were examined through ICC. The ICC value showed the degree of reproducibility of the questionnaires in repeated measurements. The high test-retest reliability could be attributed to the acceptable face and content validity of the translated questionnaire (Vyas et al., 2016). It also indicated the questionnaires were stable, in terms of the ability to reproduce measures, assuming that variables that were to be tested did not change over time (Streiner, Norman \& Cairney, 2014).

In our study, total ICC values of M-CFFQ and M-OHKAPQ showed excellent agreement between test and retest. The overall ICC value of M-CFFQ was 0.887 (95\% CI; 0.788, 0.938). As for specific domains, values of ICC ranged from 0.608 (traditional delicacies) to 0.872 (sugared cereal). The total ICC value of M-OHKAPQ was $0.807(95 \%$ CI; 0.680, 0.887). The ICC value for the Knowledge domain was 0.714 (95\% CI; $0.542,0.829)$, the Attitude domain was 0.858 (95\% CI; 0.759, 0.918) and the 
Practice domain was 0.876 (95\% CI; $0.790,0.928)$. The smaller ICC value was noted for the Knowledge domain was due to the lower agreement for the two occasions the questionnaire was administrated. It is possible that the first administration may have induced the participants to seek the right answers which then led to the changes of responses in the second administration.

\section{CONCLUSION}

The content validity and content face validity of the Bahasa Melayu version of CFFQ (M-CFFQ) and OHKAPQ (M-OHKAPQ) for parents of children aged 6-11 years old in Kota Bharu, Kelantan were satisfactorily established in the translation process. M-CFFQ and M-OHKAPQ also successfully achieved semantic, idiomatic, experiental and conceptual equivalences. They showed excellent results in test-retest reliability (ICC >0.800). Both M-CFFQ and M-OHKAPQ have been proven to be linguistically valid and reliable in this study.

This study was a first attempt to adapt the questionnaires from the original sources. Therefore, the findings could not be compared with any previous studies. Further studies on psychometric testing of M-CFFQ and M-OHKAPQ are suggested to assess other aspects of validity and reliability. The studies should be conducted in a larger Malaysian population with different ethnic variations and sociodemographic backgrounds. They could be improved with the establishment of a user's guide, which could include features such as the mode of administration, references, scoring and interpretation of the scores, to help future researchers using the questionnaires. The cariogenicity index and quantity measurement of the foods or drinks could be incorporated in $\mathrm{M}-\mathrm{CFFQ}$, to produce a more meaningful and valuable assessment for dental studies.

\section{Acknowledgement}

The authors wish to express their gratitude to all parents, school headmasters, teachers and all children who participated in this study. We wish to extend our deepest thanks to the personnel of Oral Health Programme of the Ministry of Health Malaysia, the Dean of the School of Dental Sciences of Universiti Sains Malaysia for their full support and to the Director General of Health Malaysia for permission to publish this article.

\section{Authors' contributions}

$\mathrm{RN}$, the principal investigator, conducted the study, data collection and data analysis and drafted the manuscript; $\mathrm{RH}$, undertook data analysis, interpretation of data, assisted in drafting and review of the manuscript; NAR performed the data analysis and interpretation of data, and assisted in the drafting and review of the manuscript.

\section{Conflict of interest}

The study was internally funded. The authors declare no conflict of interest.

\section{References}

Arifin WN (2015). Sample size calculator (Version 1.7.1) [Spreadsheet file]. Pusat Pengajian Sains Perubatan, Universiti Sains Malaysia, Kubang Kerian, Kelantan, Malaysia. From http:// medic.usm.my/biostat/. [Retrieved November 25 2016].

Arora A \& Evans RW (2012). Is the consumption of fruit cariogenic? Journal of Investigative and Clinical Dentistry 3(1):17-22. doi: 10.1111/j.2041-1626.2011.00076.x.

Beaton DE, Bombardier C, Guillemin F \& Ferraz MB (2000). Guidelines for the process of crosscultural adaptation of self-report measures. Spine 25(24):3186-3191.

Cheah W, Tay S, Chai S, Bong C, Baharuddin LH \& Che Jalil ZJ (2010). Oral health knowledge, attitude and practice among secondary school students in Kuching, Sarawak. Archives of Orofacial Sciences 5(1):9-16.

Chee SS, Zawiah H, Ismail MN \& Ng KK (1996). Anthropometry, dietary patterns and nutrient intakes of Malaysian estate workers. Malaysian Journal of Nutrition 2:112-126.

Cicchetti DV (1994). Guidelines, criteria, and rules of thumb for evaluating normed and standardized assessment instruments in psychology. Psychological Assessment 6(4):284. 
Epstein J, Santo RM \& Guillemin F (2015). A review of guidelines for cross-cultural adaptation of questionnaires could not bring out a consensus. Journal of Clinical Epidemiology 68(4):435-441.

Fejerskov O (2004). Changing paradigms in concepts on dental caries: consequences for oral health care. Caries Research 38(3):182191. doi: 10.1159/000077753.

Habibian M, Roberts G, Lawson M, Stevenson $R$ \& Harris S (2001). Dietary habits and dental health over the first 18 months of life. Community Dentistry and Oral Epidemiology 29(4):239-246.

Hammond A, Tyson S, Prior Y, Hawkins R, Tennant A, Nordenskiold U, Thyberg I, Sandqvist G \& Cederlund R (2014). Linguistic validation and cultural adaptation of an English version of the Evaluation of Daily Activity Questionnaire in rheumatoid arthritis. Health and Quality of Life Outcome 12(1):143.

Karthikeyan G, Manoor U \& Supe SS (2015). Translation and validation of the questionnaire on current status of physiotherapy practice in the cancer rehabilitation. Journal of Cancer Research and Therapeutics 11(1):29-36.

Laaksonen C (2012). Validation of Instrument, Child Self Assessment, Parent-Proxy Assessment and School Nursing Documentation of Health Checkups (Thesis). University of Turku, Turku, Finland.

Mani S, Aziz A, John J \& Ismail (2010). Knowledge, attitude and practice of oral health promoting factors among caretakers of children attending day-care centers in Kubang Kerian, Malaysia: A preliminary study. Journal of Indian Society of Pedodontics and Preventive Dentistry 28(2):78.

Mani SA, John J, Ismail NM \& Ping WY (2012). Early Childhood Caries: Parent's Knowledge, Attitude and Practice Towards Its Prevention in Malaysia: Citeseer.

Moynihan P \& Petersen PE (2004). Diet, nutrition and the prevention of dental diseases. Public Health Nutrition 7(1A; SPI):201-226.

Naidu R \& Davis L (2008). Parents' views on factors influencing the dental health of Trinidadian pre-school children. Community Dental Health 25(1):44-49.

NCCFN (2010). Malaysian Dietary Guidelines. National Coordinating Committee for Food and Nutrition, Ministry of Health Malaysia, Putrajaya.
Oral Health Division Malaysia (2015). National Oral Health Survey of Preschool Children 2015. Ministry of Health Malaysia, Putrajaya.

Oral Health Division Malaysia (2017). National Oral Health Survey of School Children 2017. National Health and Morbidity Survey, Ministry of Health Malaysia, Putrajaya.

Peres MA, Sheiham A, Liu P, Demarco FF, Silva AER, Assunção MC, Menezes AM, Barros FC \& Peres KG (2016). Sugar Consumption and Changes in Dental Caries from Childhood to Adolescence. Journal of Dental Research 95(4):388-394. doi: 10.1177/0022034515625907.

Petersen PE, Bourgeois D, Ogawa H, EstupinanDay S \& Ndiaye C (2005). The global burden of oral diseases and risks to oral health. Bulletin of the World Health Organization 83(9):661669.

Rathmawati A, Normastura AR, Ruhaya H \& Azizah Y (2017). Oral health knowledge, attitude and practice of parents and frequency of cariogenic food intake among their cleft children in Kelantan. Malaysian Journal of Nutrition 23(2):239-252.

Rugg-Gunn A \& Woodward M (2011). Milk and oral health. From http://www.borrowfoundation. org/assets / uploads/milk-and-oral-health.pdf. [Retrieved March 24 2017].

Ruhaya H, Nasruddin J, Marhazlinda J, AbdulRashid I, Noorliza MI \& Tambi Chik B (2012). Nutritional status and early childhood caries among preschool children in Pasir Mas, Kelantan, Malaysia. Arch Orofac Sci 7(2):56-62.

Schroth RJ, Brothwell DJ \& Moffatt ME (2007). Caregiver knowledge and attitudes of preschool oral health and early childhood caries (ECC). International Journal of Circumpolar Health 66(2):153-167.

Shinga-Ishihara C, Nakai Y, Milgrom P, Murakami K \& Matsumoto-Nakano M (2014). Crosscultural validity of a dietary questionnaire for studies of dental caries risk in Japanese. BMC Oral Health 14(1):1-8. doi: 10.1186/14726831-14-1.

Streiner DL, Norman GR \& Cairney J (2014). Health measurement scales: a practical guide to their development and use: Oxford University Press, USA

Tinanoff N \& Reisine S (2009). Update on early childhood caries since the Surgeon General's Report. Academic Pediatrics 9(6):396-403. doi: 10.1016/j.acap.2009.08.006. 
Vyas S, Nagarajappa S, Dasar PL \& Mishra P (2016). Linguistic adaptation and psychometric evaluation of original Oral Health Literacy-Adult Questionnaire (OHL-AQ). Journal of Advanced Medical Education and Professionalism 4(4):163-169.

Walter S, Eliasziw M \& Donner A (1998). Sample size and optimal designs for reliability studies. Statistics in Medicine 17(1):101-110.

Watt RG, Harnett R, Daly B, Fuller SS, Kay E, Morgan A, Munday P, Nowjack-Raymer R \& Treasure ET (2004). Oral Health Promotion: Evaluation Toolkit. Stephen Hancocks Ltd, London.
WHO (2001). Process of translation and adaptation of instruments. From http://www.who. int/ substance_abuse/research_tools / translation / en/ [Retrieved November 24 2016].

Zahara A, Fashihah M \& Nurul A (2010). Relationship between frequency of sugary food and drink consumption with occurrence of dental caries among preschool children in Titiwangsa, Kuala Lumpur. Malaysian Journal of Nutrition 16(1):83-90.

Zulkifli MR, Tin-Oo MM \& Saddki N (2015). Crosscultural adaptation and validation of oral health literacy instrument for use in Malaysia (Master Dissertation). Universiti Sains Malaysia. 